\title{
Baroque
}

\section{Résumé de la communication d'Anne Viguier}

\section{Anne Viguier}

\section{OpenEdition}

\section{Journals}

Édition électronique

URL : http://journals.openedition.org/baroque/524

DOI : 10.4000/baroque.524

ISSN : 2261-639X

\section{Éditeur :}

Centre de recherches historiques - EHESS, Éditions Cocagne

Édition imprimée

Date de publication : 1 mai 1980

ISSN : 0067-4222

\section{Référence électronique}

Anne Viguier, "Résumé de la communication d'Anne Viguier », Baroque [En ligne], 09-10 | 1980, mis en ligne le 15 mai 2013, consulté le 21 avril 2019. URL : http://journals.openedition.org/baroque/524 : DOI : 10.4000/baroque.524

Ce document a été généré automatiquement le 21 avril 2019

(c) Tous droits réservés 


\title{
Résumé de la communication d'Anne Viguier
}

\author{
Anne Viguier
}

\section{Intervention}

1 Ses travaux sur le baroque entre 1580 et 1640 et ses recherches sur la signification du discours baroque en peinture, sculpture, poésie et théologie. L'enquête porte sur le discours, son sens, ses structures, les mentalités qu'il révèle, en particulier dans, les régions occitanes.

2 Le baroque est promotion du logos, comparaison et raison. En poésie, d'Aubigné illustre en particulier le délire rationnel du baroque dans les cent premiers vers des Tragiques. Le discours théologique cherche à comprendre un ordre dans une succession d'images fantastiques. Discours théologique et poésie révèlent un code, une convention. Les œuvres en prose : Montaigne, La Noue, fabriquent un discours d'apparence désordonnée, mais qui possède cependant une construction logique.

3 Comment aborder les structures dynamiques de ces textes? À la fin du Xvi siècle et au début du XVII ${ }^{\mathrm{e}}$, les questions d'astrophysique, le centre du monde, la place de la terre sont le sujet des grandes oppositions; les conflits sont théologiques et politiques. Quelles figures surgissent dans les textes littéraires au cours de ces débats, sur la conception du monde ? L'image du cercle en plan, qui existe dès le Moyen Âge, semble vite désuète ; en revanche, à la fin du Xvi ${ }^{\mathrm{e}}$ siècle, avec Képler, surgissent de nouvelles images: volume, sphère, ellipse. Les thèmes du miroir et du reflet, générateurs à la fois de vérité et de tromperie, renvoient aussi au cercle de Marguerite de Navarre à la fin du XvI ${ }^{\mathrm{e}}$ siècle.

$4 \quad$ Il s'agit d'étudier le fonctionnement de ces métaphores dans le Quart Livre de Rabelais et les poèmes de Goudouli.

5 Dans la littérature héroïque, à l'époque baroque, les structures concentriques renvoient à un monde bien construit avec un «centre plein». Dans les textes carnavalesques, 
apparaissent, au contraire, l'univers décentré et le monde à l'envers. Ces décrochages se retrouvent chez Goudouli et Rabelais; Montaigne utilise les deux structures dans le chapitre sur les vers de Virgile.

6 Les structures de l'analogie font déborder le baroque sur la Renaissance ; la « branloire pérenne", les textes de Montaigne sur la boiteuse renvoient à un univers postcopernicien.

\section{Débat Krynen, Wagner, Hallyn, Auzias, Duval-Virth, Mansau, Martinon}

7 Les théologies de François de Sales et de Calvin ne semblent pas différentes si nous recherchons les codes et les constructions; en effet, les structures d'emploi et de réemploi ne peuvent plus être distinguées dans la théologie du baroque qui s'appuie sur l'homme. Calvin, Luther, François de Sales reprennent la même structure, mais ils vont dans des sens opposés. Ces esprits différents emploient saint Paul dans des architectures aux significations différentes. Pour distinguer Calvin et saint François de Sales, ne faut-il pas séparer la pensée et son contenu des structures?

8 Le baroque occitan apparaît-il comme la transcription d'une mentalité générale qui préside aux relations entre modèle latin et langues vernaculaires? Pour Goudouli et Mainard, les matériaux, les codes, les images et les mots qui évoquent Henri IV sont identiques ; la langue et la syntaxe créent cependant une différence. Du pétrarquisme au gongorisme, l'utilisation de la langue est différente. En Occitanie, le modèle latin demeure, quoique l'occitan possède un statut de langue.

9 - LAFAY : Comment remettre en cause la conception du baroque, telle est la question que je pose à la fin des débats de cette journée. Pour le littéraire, il ressort un aspect négatif : il ne peut utiliser le concept de baroque. Il faut voir aussi un éclairage positif : le baroque, type relationnel, ne peut être atteint par une analyse séparée des différentes spécialités.

10 Les problèmes du baroque doivent donc se situer dans l'optique d'une linguistique généralisée; il est nécessaire, pour comprendre les œuvres de connaître les théories contemporaines, mais ce point de vue est aussi discutable, car à cette époque, la théorie esthétique est plus pauvre que les textes. D'autre part, aujourd'hui, les progrès de la méthodologie permettent une meilleure approche. À partir des théories contemporaines et des concepts modernes, l'exégète donne un meilleur sens aux textes.

11 - WAGNER : La connaissance des théories contemporaines de l'œuvre unie à l'expérience émotive et psychologique est indispensable.

Faut-il se garder de projeter dans le passé nos problèmes actuels ou faut-il agir avec empathie?

12 - HALLYN : La théorie n'est pas neutre: approche des apparences par Jean Rousset, approche structuraliste par Gérard Genette... L'identification peut conduire à un mirage.

13 - KRYNEN: La méthodologie rejoint le problème du logos: comment analyser les références au logos? Dieu permet à l'analyste de révéler la spécificité mystique du texte théologique ; les mystiques baroques sont des exégètes qui dérivent hors de la mystique.

14 - WAGNER : Dieu se trouve-t-il dans l'environnement socio-culturel? En approchant le texte dans un contexte uniquement historique, le chercheur mutile la partie mystique. 
Comment intégrer la mystique dans l'histoire? Fait-on l'histoire de la théologie sur un fond eschatologique?

15 - KRYNEN : Le problème des théologiens de l'époque est le suivant: que devient le Christ si on le rationalise? Les mystiques baroques vivent le drame du mystique qui cesse de l'être.

16 - MARTINON : La coupure fondamentale se trouverait donc entre culture absolue et culture relative.

17 - HALLYN : On recherche l'homme signifiant, la relation entre l'homme et Dieu.

18 - KRYNEN : La réponse donnée au Christ est celle de l'homme signifié. La théologie du baroque est drame.

\section{Discussion entre Krynen, Martinon, Auzias, Wagner, Castan}

19 Peut-on comprendre la culture baroque en dehors de la théologie et de la mystique? L'approche structuraliste n'oblige nullement à participer aux croyances, mais peut-on comprendre une croyance de l'extérieur? Entre 1560 et 1650, les ouvrages théoriques d'iconologie ou autres sont des faits historiques qu'il faut obligatoirement utiliser. Ces textes utilisent une idéologie platonicienne qu'il faut accepter pour les comprendre. Les dangers de la fausse objectivité et du néo-positivisme sont à éviter. La démarche scientifique se trouve dans la coupure avec les préjugés idéologiques; les perspectives méthodologiques semblent plus importantes que les philosophies dans l'approche du logos baroque.

\section{AUTEUR}

\section{ANNE VIGUIER}

Maître-Assistant de Littérature française Université Toulouse Le Mirail 